\title{
Diseño e Innovación Social como herramienta para el cumplimiento de Objetivos de Desarrollo Sostenible.
}

\author{
Mtra. Blanca Ivette Duarte Álvarez ${ }^{a}$, Dr. David Cortés Sáenz ${ }^{\text {b }}$ \\ ${ }^{a}$ Maestra en Diseño de Producto por ELISAVA. Adscrita al Instituto de Arquitectura, Diseño y Arte. \\ Universidad Autónoma de Ciudad Juárez. México. blanca.duarte@uacj.mx ${ }^{\text {b }}$ Doctor en Innovación \\ Tecnológica por la Universidad Politécnica de Catalunya Adscrito al Instituto de Arquitectura, Diseño \\ y Arte. Universidad Autónoma de Ciudad Juárez. México. david.cortes@uacj.mx
}

\begin{abstract}
Resumen
El diseño para la innovación social se enfoca en la creación e implementación de soluciones que fomentan mejores condiciones sociales, proyectadas en comunidades, ciudades o regiones a través de la innovación, la conciencia social y la equidad. Los seres humanos innovan cuando enfrentan un problema o reto. Problemas sociales complejos identificados como metas en los Objetivos de Desarrollo Sostenible (ODS) pueden ser mitigados a través de soluciones que se obtienen en procesos de diseño, en ese sentido el diseñador se convierte en un potencial agente de cambio. Las personas de la comunidad para la cual se diseña pueden ser incorporadas al proceso de diseño por medio de sesiones de co-creación y métodos de diseño participativo, esto puede traer un impacto positivo en las soluciones proyectadas. Éste artículo está basado en una revisión de literatura especializada, y el uso de la metodología Design Research Methodology DRM de Blessing y Chakrabarti (2009); y aborda como los procesos de diseño y la innovación contribuyen a identificar y resolver problemas que aquejan a la sociedad, identificados como metas en los Objetivos de Desarrollo Sostenible.
\end{abstract}

Palabras clave: Innovación Social, Diseño Participativo, Co-creación, Codiseño, $O D S, D R M$

\section{Introducción}

El diseño en virtud de su propia naturaleza es una actividad social, sus objetivos pueden ser identificados de acuerdo a diversos enfoques, como describe Heller (2018), mientras que en los enfoques tradicionales de diseño se aspira a resolver una necesidad a través de una 
idea transformada en la próxima estrategia o producto genial; en el enfoque de Diseño para la Innovación Social se busca crear una condición futura que permita resolver retos sociales a partir de la participación y compromiso del diseñador, la propia comunidad; así como la generación de productos, servicios, estrategias o métodos que fomenten una nueva situación social en ese entorno.

El Diseño para la innovación social es definido por Manzini (2013) como un proceso de cambios que emerge de la combinación creativa de los recursos existentes (del capital social al patrimonio histórico, de la artesanía tradicional a la tecnología avanzada y accesible).

La innovación social, de acuerdo a Ortiz Nicolás $(2019$, p.15) representa una alternativa para lidiar con algunos de los retos emergidos de las prácticas actuales, a la par que busca un fin específico: el bien social.

A medida que el mundo continúa experimentando cambios, sus retos se multiplican y se tornan más intrincados, los desafíos a los que la sociedad se enfrenta, son muy diversos, por mencionar algunos: desafíos sociales, económicos o ambientales; el diseño tiene el potencial necesario para desempeñar un papel destacado como detonante y soporte del cambio social, y convertirse por tanto, en diseño para la innovación social (Manzini, 2015).

El diseño para la innovación social toma acción a través de numerosos campos del diseño, como la arquitectura, el urbanismo, el diseño gráfico, el diseño industrial y en muy diversos entornos; desde el desarrollo de iniciativas para hacer frente a catástrofes como el sismo en la Ciudad de México en septiembre 2018, facilitar a través de un producto el almacenamiento y transporte de agua en varias comunidades críticas de Venezuela en 2019 o el desarrollo de una estación de reciclaje que explora el manejo de residuos y la sostenibilidad desde una perspectiva holística en Malmö, Suecia.

\section{Objetivos}

El propósito de este documento es explorar la posibilidad que el Diseño para la Innovación Social tiene para transformar las condiciones sociales del entorno en el que se desarrolla, también busca reflexionar cómo los métodos y herramientas del Diseño Participativo y Cocreación facilitan los procesos de Diseño para la Innovación social, así como identificar cómo los procesos de diseño y la innovación contribuyen a identificar y resolver retos como los Objetivos de Desarrollo Sostenible. 


\section{Fundamentos Teóricos}

\subsection{Innovación a través del Diseño}

La innovación se entiende como social en la medida en que la sociedad se reorganiza para resolver sus problemas de manera innovadora; En este caso, la innovación está principalmente vinculada a cambios en el comportamiento individual hacia formas de vida más sostenibles (Freire, K., Borba G. \& Diebold L., 2011).

Según Kumar (2013) en la innovación en diseño, lo más importante es crear innovaciones que se adapten bien a los usuarios. El enfoque cambia de los productos a las personas que los usan, y a lo que esas personas hacen: sus comportamientos, actividades, necesidades y motivaciones.

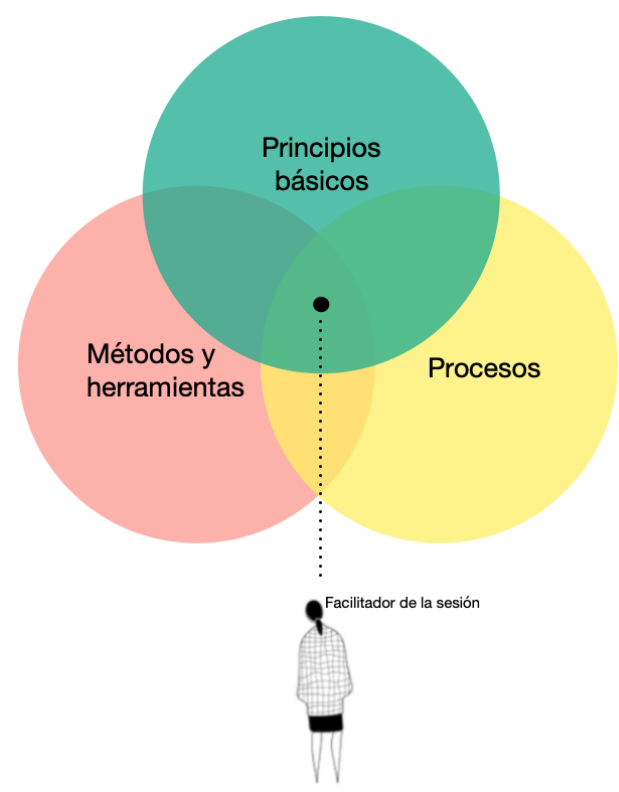

Fig 1: Componentes del Diseño Participativo (Adaptada de Designing with people 2016)

\subsection{Diseño Participativo como impulsor del proceso creativo}

De acuerdo a Maase, S \& Dorst, K (2006) los principios, herramientas y métodos de diseño participativo se utilizan en el desarrollo de productos, tecnologías o instituciones sociales. El objetivo de los métodos de diseño participativo es desarrollar una mayor capacidad de respuesta a las necesidades humanas, por lo tanto, incorporar éstos métodos al grupo de personas de la comunidad (en la cual se proyecta la solución de diseño) e involucrar a 
Diseño e Innovación Social como herramienta para el cumplimiento de Objetivos de Desarrollo Sostenible.

quienes serán los usuarios de manera más profunda en el proceso, permite que los requerimientos se incorporen mejor y por tanto sus necesidades se satisfagan con mayor precisión.

Desde el enfoque del D-Lab en el Instituto Tecnológico de Massachusetts (MIT), los procesos de diseño participativo no solo producen productos alineados con las necesidades de las personas, sino que también desarrollan habilidades y confianza en los participantes, cuando un participante diseña algo para sí se convierte de usuario a un innovador/solucionador de problemas, de manera similar Manzini (2015, p.47) plantea dos polos para aquellos que diseñan: el diseño difuso que es puesto en marcha por "inexpertos" que hacen uso de su capacidad natural para el diseño, y el diseño experto personas formadas para actuar como tales de manera competente y se proponen a sí mismos como profesionales de la disciplina.

\subsection{Innovación a través de Co-creación}

Sanders, E. y Simons, G. (2009) definen la co-creación como un acto de creatividad colectiva que es experimentado y realizado conjuntamente por un grupo de personas. El codiseño es creatividad colectiva que se aplica en todo el proceso de diseño. Esto significa que el co-diseño es una instancia específica de la co-creación.

Una sesión de co-creación puede ser una excelente oportunidad para ayudar al equipo de diseño a comprender mejor el reto al que se está enfrentando, obtener comentarios sobre propuestas, incluso a guiar a personas para que se integren al equipo y participen de una manera más activa en el proceso de diseño.

La co-creación sostiene que los usuarios tienen el conocimiento de sus propias necesidades y pueden ser creativos si se les facilita y motiva a ser, sin embargo, contrariamente al diseño participativo (Spinuzzi, C. 2005 citado en Nielsen L. 2011) las innovaciones del codiseño podrían no conducir a artefactos que los participantes usen para sí mismos, se identifican dos planteamientos de co-diseño en productos; en el primero se conciben los productos fuera del contexto, comúnmente en países desarrollados (o en vías de desarrollo) y luego se implementan en comunidades más vulnerables y el segundo, desde una perspectiva evidentemente más social, donde las personas de la propia comunidad para la que se diseña participan de manera activa, lo que permite una mayor implicación.

Dott 07 es un programa que surge a través del Design Council, para mejorar la vida de las personas a través del diseño; fundamentándose en desarrollo de proyectos de innovación a través de una metodología propia, usada en sesiones de co-creación, el rediseño de servicios públicos y comunitarios, publicaciones, exhibiciones, John Thackara (2008) 
participó en la creación de éste programa, su metodología consiste en cinco fases: Diagnóstico, Co-descubrimiento, Co-diseño, Co-desarrollo y Soluciones.

\subsection{Objetivos de Desarrollo Sostenible hacia el 2030}

De acuerdo a el Programa de las Naciones Unidas para el Desarrollo (PNUD), los Objetivos de Desarrollo Sostenible, se establecieron en 2015 como un plan de acción para poner fin a la pobreza, proteger el planeta y garantizar que todas las personas gocen de paz y prosperidad para 2030, en su manifiesto afirman que se comprometen a acelerar el progreso de los países más atrasados, que el desarrollo buscará equilibrar la sostenibilidad social, económica y medio ambiental.

De acuerdo a como integran los ODS, consideran que si se interviene en un área, ésta seguramente repercutirá también en otras áreas, el PNUD reconoce que para cumplir con éstos objetivos es necesario creatividad, conocimiento, tecnología y recursos financieros de toda la sociedad para alcanzar los ODS, teniendo en cuenta éstos aspectos podemos reconocer que los métodos, herramientas y metodologías que ofrece el Diseño para la Innovación Social, el Diseño Participativo y la Co-creación pueden ser recursos que faciliten el cumplimiento de éstos ODS.

\section{Objetivos de DesarrolloSostenible}

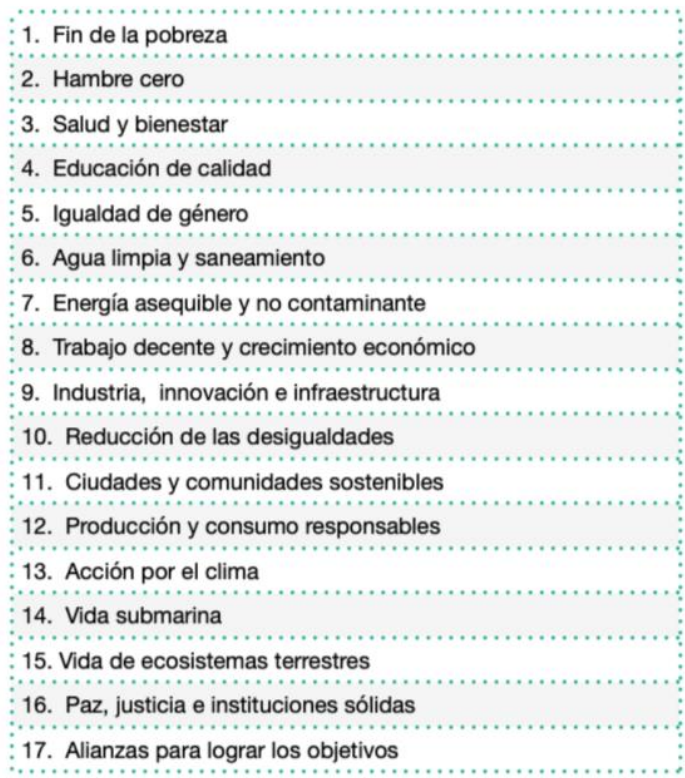

Fig 2: Objetivos de Desarrollo Sostenible. Elaboración propia 2020.

Fuente: (http://www.un.org) 
Diseño e Innovación Social como herramienta para el cumplimiento de Objetivos de Desarrollo Sostenible.

\section{Metodología}

El presente artículo se ha desarrollado a partir una revisión de literatura especializada y de la metodología Design Research Methodology (DRM) de Blessing y Chakrabarti (2009), DRM la describen sus autores como una metodología genérica de investigación de diseño que vincula las preguntas de investigación y brinda apoyo para abordarlas de manera sistemática, el proyecto propone las siguientes etapas:

- Definición de Criterios: Genera evidencia de que el campo de investigación es válido y se especifica el objetivo de la investigación.

- Estudio Descriptivo I: Se cuenta con un objetivo y enfoque claros, se revisa literatura en busca de más factores que influyan, identifica los principales desafíos en la investigación y determina en qué factores debe centrarse la investigación continua.

- Estudio prescriptivo: Se centra en mejorar la calidad de la definición del problema como el factor más prometedor para abordar, se comienza el desarrollo sistemático de un soporte para mejorar la calidad de la definición del problema, puede ser el uso de herramientas o software que apoyen al proceso.

- Descripción Estudio II (DS-II): Evaluación del apoyo desarrollado en comparación con el objetivo de la investigación. investiga el impacto del apoyo y su capacidad para darse cuenta de la situación deseada. Emprenden dos estudios empíricos para comprender el uso real del soporte.

En el presente documento, la metodología DRM se inicia con la primer etapa Definición de Criterios, continuando con un análisis de literatura como principio de la etapa Estudio Descriptivo I que se utilizará para el desarrollo de la investigación.

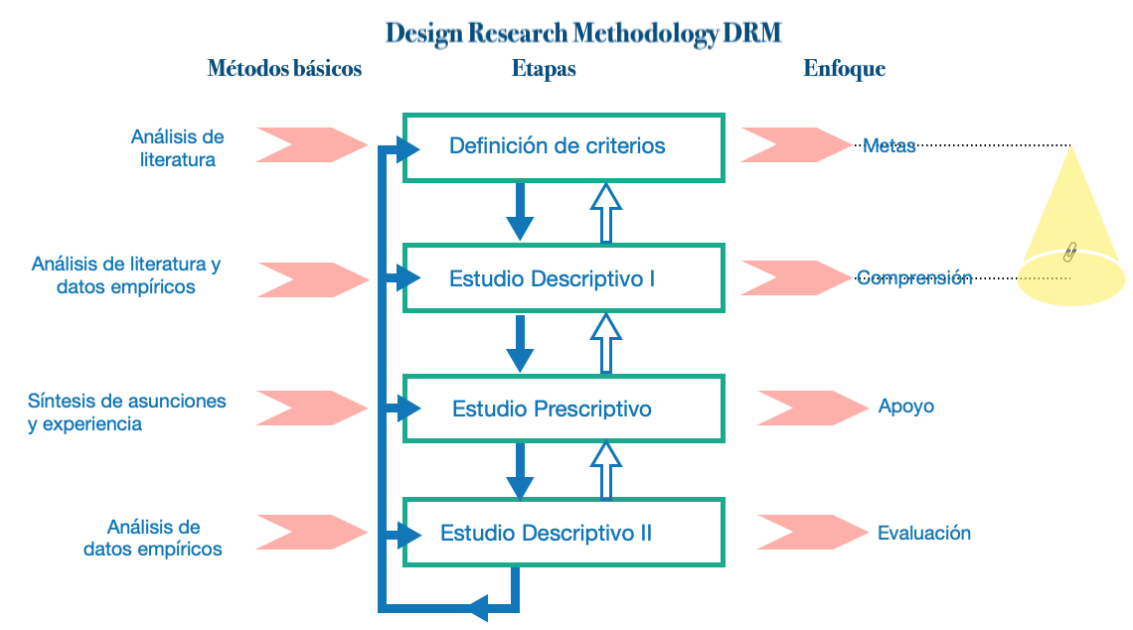

Fig 4: Design Research Methodology. (Adaptado de Blessing y Chakrabarti (2009) 
En la fig. 4 no muestra las muchas iteraciones y la ejecución paralela de etapas que son parte de la realidad. Tampoco muestra que el punto de partida puede estar en cualquiera de las etapas y que es posible, en un proyecto individual, concentrarse solo en una o dos etapas. (Blessing, L. \& Chakrabarti, A, 2009, p. 17).

\section{Conclusiones}

Dentro de éste artículo y de acuerdo a la metodología seleccionada se decidió tomar por etapa inicial la definición de criterios básicos para contextualizar la propia disciplina del Diseño para la Innovación Social, que si bien, tiene similitudes con el área de Diseño de Producto tiene una importante diferencia: el Diseño para la Innovación Social busca promover el bien social a través de innovar para lograr afrontar retos que la sociedad enfrenta.

Se proponen métodos de Diseño Participativo y Co-creación para lograr los propósitos del Diseño para la Innovación Social, ambos métodos podrían utilizarse de manera indistinta; eligiendo la mejor herramienta para cada actividad, además éstos métodos comparten características en común; la más importante es que de una manera u otra la figura del usuario esté presente en el desarrollo del método.

Se puede afirmar que el diseño participativo al incorporar a las personas que finalmente serán los usuarios permite obtener soluciones de diseño más precisas que se adaptan mejor a las necesidades del usuario; por otro lado podemos observar que los participantes no sólo desarrollan habilidades de inspiración o ideación, sino que pueden llegar a desarrollar confianza en sí mismos y actitudes positivas, que de una u otra manera pueden beneficiar a la propia comunidad.

Como ya se ha mencionado, la co-creación es un acto de creatividad colectiva, y el codiseño una vertiente específica de la co-creación, en éste método se plantea de dos maneras: la primera, diseñar y desarrollar los artefactos en un lugar para implementarlos en otro, -esto permite diferenciarlo del Diseño Participativo- ó desde una perspectiva más social y por tanto de mayor interés para el Diseño para la Innovación Social, realizar las sesiones de co-diseño en el lugar donde se encuentran sus usuarios.

Se plantea que los Objetivos de Desarrollo Sostenible -metas promovidas por el Programa Nacional de las Naciones Unidas para garantizar el bienestar de las personas del presente al 2030-, pueden cumplirse a través de fomentar la creatividad, transmitir el conocimiento, o desarrollar tecnología; estrategias acordes con los métodos propuestos de Diseño Participativo y Diseño para la Innovación Social. 
Diseño e Innovación Social como herramienta para el cumplimiento de Objetivos de Desarrollo Sostenible.

\section{Referencias}

Blessing, L and Chakrabarti, A. (2009). DRM, a Design Research Methodology. London: Springer, 2009.

Freire, K. , Borba G. \& Diebold L. (2011). Participatory Design as an Approach to Social Innovation. Brasil: Universidade do Vale do Rio dos Sinos São Leopoldo.

Kumar, V. (2013). 101 Design Methods. New Jersey: John Wiley \& Sons.

Heller, C. (2018). The Intergalactic Design Guide. Washington DC: Island Press

Maase S \& Dorst K..(2006). Chapter 22 Co-creation: A way to reach sustainable social innovation?. Proceedings: Changes to Sustainable Consumption (296). Copenhagen, Denmark: Workshop of the Sustainable Consumption Research Exchange.

Manzini Ezio. (2013). Making Things Happen: Social Innovation and Design. Design Issues, 30, $57-$ 66. mitpressjournals.org

Manzini Ezio. (2015). Cuando todos diseñan. Madrid: Experimenta Editorial.

Martin, B \& Hanington B. (2012). Universal Methods of Design. United States of America: Rockport Publishers.

Nielsen, L. (2011). Personas in Co-creation and Co-design. Copenhagen: IT University of Copenhagen.

Ortiz Nicolás, J. (2019). Innovación social y diseño. Ciudad de México: Centro de Investigaciones de Diseño de la UNAM. Universidad Nacional Autónoma de México.

Sanders, E.B.-N. \& Stappers P.J. (2008). Co-creation and the new landscapes of design. Design and the Arts. Volume 4, 2008 - Issue 1: Design Participation.

Thackara John. (2008). Transforming public services. Innovation by design in public services, p. 2630. Design Council: London. 